\title{
Handling the Temperature Effect in Vibration Monitoring: Two Subspace-Based Analytical Approaches
}

\author{
Michèle Basseville ${ }^{1}$; Frédéric Bourquin²; Laurent Mevel ${ }^{3}$; Houssein Nasser ${ }^{4}$; and Fabien Treyssède ${ }^{5}$
}

\begin{abstract}
The dynamics of most civil engineering structures is affected by the ambient temperature. This raises the issue of discriminating changes in modal parameters due to damage from those due to such effects. A statistical parametric damage detection algorithm based on a null space residual associated with output-only subspace identification and a $\chi^{2}$ test built on that residual has been designed by some of the writers. The purpose of this paper is to propose two extensions of this detection method which account for the temperature effect. The first extension uses a thermal model for deriving a temperature-adjusted null space. The second extension exploits the thermal model together with a statistical nuisance rejection technique. Both methods are illustrated on a laboratory test case within a climatic chamber.
\end{abstract}

DOI: 10.1061/(ASCE)0733-9399(2010)136:3(367)

CE Database subject headings: Vibration; Damage; Assessment; Temperature effects; Analytical techniques; Structural dynamics.

\section{Introduction}

Detecting and localizing damages for monitoring the integrity of structural and mechanical systems are topics of growing interest due to the aging of many engineering constructions and structures and to increased safety norms. Automatic global vibration-based structural health monitoring (SHM) techniques have been recognized to be useful alternatives to visual inspections or local nondestructive evaluations performed manually (Pandey and Biswas 1994; Natke and Cempel 1997; Farrar et al. 2001; Catbas et al. 2004; Fritzen 2005; Alvandi and Cremona 2006; Gao et al. 2007; Zang et al. 2007). When processing vibration data (recorded with, e.g., accelerometers), a major focus is on extracting modal parameters (modal frequencies and associated damping values and mode shapes) which characterize the dynamics of the structure. It has been widely acknowledged that changes in frequencies bear useful information for damage detection, and information on changes in mode shapes is mandatory for performing damage localization.

It is also widely recognized that the dynamics of large civil structures, such as bridges, is significantly affected by the ambient temperature and other environmental effects, such as changes in

${ }^{1}$ IRISA, Campus de Beaulieu, 35042 Rennes Cedex, France (corresponding author); and CNRS. E-mail: michele.basseville@irisa.fr

${ }^{2}$ Division for Metrology and Instrumentation, LCPC, 58, bd Lefebvre, 75732 Paris Cedex 15, France. E-mail: bourquin@lcpc.fr

${ }^{3}$ Centre Rennes-Bretagne Atlantique, iNRiA, Campus de Beaulieu, 35042 Rennes Cedex, France. E-mail: laurent.mevel@inria.fr

${ }^{4}$ Centre de Recherche Public Henri Tudor, 29, Avenue John F. Kennedy, Kirchberg L-1855, Luxembourg. E-mail: houssein.nasser@ tudor.lu

${ }^{5}$ Division for Metrology and Instrumentation, LCPC, Route de Pornic, BP 4129, 44341 Bouguenais Cedex, France. E-mail: fabien.treyssede@ lcpc.fr

Note. This manuscript was submitted on January 3, 2008; approved on October 26, 2009; published online on February 12, 2010. Discussion period open until August 1, 2010; separate discussions must be submitted for individual papers. This paper is part of the Journal of Engineering Mechanics, Vol. 136, No. 3, March 1, 2010. @ASCE, ISSN 0733-9399/ 2010/3-367-378/\$25.00. loads, boundary conditions, moisture, wind, etc. Typically temperature variations have a major effect on the stiffness and thus on the dynamics of the structure, whereas the humidity mainly affects its mass. Moreover, changes in modal parameters due to environmental effects can be larger than changes due to damages (Moorty and Roeder 1992; Cornwell et al. 1999; Alampalli 2000; Rohrmann et al. 2000; Peeters and De Roeck 2001; Kullaa 2003). This raises the issue of discriminating between changes in modal parameters due to damages and changes in modal parameters due to environmental effects. Handling environmental effects has recently become a major research issue for SHM. A number of methods have been designed for addressing those issues for different civil engineering application examples (Peeters et al. 2001; Golinval et al. 2004; Kullaa 2004; Steenackers and Guillaume 2005; Yan et al. 2005a,b).

Typical damage indicators used for SHM include modes, mode shape curvatures, transfer functions, autoregressive (AR) model coefficients, stiffness or flexibility matrix coefficients, etc. Those indicators generally result from the processing of measured data (identification) and depend on both the structural parameters and the environmental parameters. Most model-based approaches to the handling of the environmental parameters, and especially the temperature effect, involve a model of the effect of the temperature on the eigenfrequencies (modes) or other damage indicators. For example, an analytical model of the temperature-induced movements and associated stresses in a bridge has been proposed in Moorty and Roeder (1992), an AR model has been used for the temperature effect in Peeters and De Roeck (2001), and a linear adaptive filter has been proposed in Sohn et al. (1999).

The investigation performed for the large-scale Alamosa Canyon Bridge in New Mexico in Sohn et al. (1999) involves a linear adaptive filter. It has been found that the fundamental frequency has been modified by about $5 \%$ within a 1-day monitoring period, and this frequency is correlated with the temperature difference through the bridge deck. A simple linear filter, taking two time and two spatial temperature measurements as its inputs, has been used for modeling the variability of the frequencies over the day. A regression model has also been used for estimating the confidence interval of the frequencies for a new temperature profile. In 
Rohrmann et al. (2000), the environmental effects on the eigenfrequencies identified on the West End Bridge in Berlin, Germany, has been investigated for over a 3-year period. A thermomechanical model describing the temperature effect has been proposed. A frequency shift proportional to the mean temperature of the bridge and to the temperature gradient has been observed, and the higher order modes have been found to be more affected by the temperature effect than the lower order modes. This approach requires the measurement of the temperature over the whole bridge at any time, which is difficult to transpose when it comes to handling other environmental effects. In Peeters and De Roeck (2001), a regression analysis of the identified frequencies over the temperature has been performed for the Z24 Bridge in Switzerland. A series of damages has been realized. The relation between the frequencies and the temperature has been found to be linear for positive temperatures (in ${ }^{\circ} \mathrm{C}$ ) and nonlinear for negative ones due to the variability of the asphalt elastic moduli, which significantly affects the bridge stiffness (Wood 1992). An AR model has been identified using the data associated with positive temperatures only. The prediction error, used as a damage indicator, succeeded in detecting the damage based on the first three frequencies.

In Kullaa (2003), factor analysis is used for deriving a relationship between the modes and the temperature. Basically factor analysis considers that the environmental effects are nonobservable and statistically independent variables, and performs a decomposition of the correlation matrix of monitored features. The damage indicator is a factor independent from the temperature (or the other environmental effects). This technique has been used for a crane vehicle, for which the five first eigenfrequencies compose the damage indicator. The method proposed in Vanlanduit et al. (2005) is of a similar kind. Two approaches are proposed in Deraemaeker et al. (2008). The first one consists in using modal filters for extracting damage indicators insensitive to the environmental parameters; the second consists in using factor analysis for eliminating the environmental effects from the damage indicator vector without measuring those effects; see also Kullaa (2004), Vanlanduit et al. (2005), and Yan et al. (2005a). The damage indicators are the eigenfrequencies, the mode shapes, and the Fourier transform of the output of modal filters used for reducing data measured with a large sensor array. The application example is a numerical model of a simulated bridge. The sensitivity of the damage indicators to noise levels, damages, and temperature gradients has been investigated for both approaches. The results from Kullaa (2003) and Deraemaeker et al. (2008) indicate that a factor analysis may help in performing damage detection without measuring the environmental parameters, provided that the dimension of the nonobservable vector is smaller than the dimension of the damage indicator vector.

A damage detection and localization method based on a subspace residual and $\chi^{2}$-type global and sensitivity tests has been proposed in Basseville et al. (2000, 2004) and successfully experimented on a variety of test cases (Mevel et al. 1999, 2003a,b; Peeters et al. 2003; Basseville et al. 2003). A subspace-based damage detection method inspired from that method has been proposed in Fritzen et al. (2003) for handling the temperature effect. The main idea consists in saving different reference signatures identified under different temperature conditions, and in applying the subspace-based detection algorithm to new data with a reference modal parameter updated according to the actual temperature value. This technique has been applied to a composite plate within a furnace with controlled temperature. The test values have been shown to remain nearly unchanged for safe condition under different temperature levels and to be highly sensitive to structural damages. However, this approach is limited by the number of reference modal signatures available at different temperatures.

The purpose of this paper is to propose two different extensions of the subspace-based damage detection algorithm in Basseville et al. (2004), which address the above issues for handling the temperature effect, and to investigate their relevance when applied to a laboratory test case. The rationale for the two extensions is the following. The subspace-based residual involves the null space of a matrix (observability matrix) built on reference modes and mode shapes identified on the safe structure at a specific and known temperature. When processing data that will be recorded later at a different temperature that is also assumed to be known, it is mandatory to update the null space to account for possible modal changes due to temperature effects. This is achievable either by updating the stiffness matrix, thanks to a thermal model, and computing the associated modes and mode shapes, or by updating directly the modes and mode shapes using a semianalytic approach. This is the first extension. The second extension handles the temperature effect as a nuisance parameter. A model of the temperature effect developed by some of the writers is used together with a statistical nuisance rejection approach, for deriving an extended $\chi^{2}$ test performing the rejection of the temperature effect seen as a nuisance for monitoring.

The paper is organized as follows. First, the key components of the subspace-based vibration monitoring algorithms are summarized. Second, an analytical model of the temperature effect on the modal properties is described for an Euler-Bernoulli beam, and analytical expressions for thermal sensitivities of the modal parameters are provided. Then, the handling of the temperature effect with the subspace-based approach is investigated and the two methods of temperature-adjusted subspace detection and temperature rejection, respectively, are proposed. Next, a laboratory test case is described, and numerical results obtained with these two new algorithms are reported. Finally, some conclusions are drawn.

\section{Subspace-Based Detection}

Some of the writers have advocated a subspace-based algorithm for modal monitoring. The key components of this algorithm are now recalled.

\section{Subspace-Based Residual}

It is well known (Juang 1994; Ewins 2000) that the continuoustime model of interest for vibration-based structural analysis and monitoring is of the form

$$
M \ddot{\mathcal{Z}}(t)+C \dot{\mathcal{Z}}(t)+K \mathcal{Z}(t)=\mathbf{v}(t)
$$

where $M, C, K=$ mass, damping, and stiffness matrices, respectively; $\mathcal{Z}(t)=$ displacement vector at time $t$; and the unmeasured input force $\mathbf{v}(t)=$ stationary white noise. [In case of colored input excitation, the corresponding poles are generally highly damped and can easily be separated from structural modes. The nonstationary case is analyzed in Benveniste and Mevel (2007).] Moreover, the modes $\mu$ and mode shapes $\Psi_{\mu}$ are solutions of

$$
\operatorname{det}\left(\mu^{2} M+\mu C+K\right)=0, \quad\left(\mu^{2} M+\mu C+K\right) \Psi_{\mu}=0
$$

It is also well known that, because of Eq. (1), vibration-based structural monitoring boils down to the problem of monitoring the 
eigenstructure of the state transition matrix $F$ of a discrete-time linear dynamic system

$$
\left\{\begin{array}{c}
\boldsymbol{X}_{k+1}=F \boldsymbol{X}_{k}+\boldsymbol{V}_{k+1} \\
\boldsymbol{Y}_{k}=H \boldsymbol{X}_{k}
\end{array}\right.
$$

namely the pair $\left(\lambda, \boldsymbol{\phi}_{\lambda}\right)$ solution of

$$
\operatorname{det}(F-\lambda I)=0, \quad(F-\lambda I) \boldsymbol{\phi}_{\lambda}=0
$$

Actually, matrix $F$ is related to the physical parameters $M, C, K$ through

$$
F=e^{\tau \mathcal{L}}, \quad \mathcal{L}=\left[\begin{array}{cc}
0 & I \\
-M^{-1} K & -M^{-1} C
\end{array}\right]
$$

where $\tau=$ sampling interval. The relation to the continuous-time eigenstructure (2) is $\lambda=e^{\tau \mu}$ and $\boldsymbol{\varphi}_{\lambda}=H \boldsymbol{\phi}_{\lambda}$ contains the observed components of $\Psi_{\mu}$. Let the $\left(\lambda, \varphi_{\lambda}\right)$ 's be stacked into

$$
\boldsymbol{\theta}=\left(\begin{array}{c}
\boldsymbol{\Lambda} \\
\operatorname{vec} \Phi
\end{array}\right)
$$

where $\Lambda$ =vector whose elements are the $\lambda$ 's; $\Phi=$ matrix whose columns are the $\varphi_{\lambda}$ 's; and vec $=$ column stacking operator.

It is assumed that a reference parameter $\boldsymbol{\theta}_{0}$ is available, identified on data recorded on the reference (undamaged) system. Given a new data sample, the detection problem is to decide whether it is still well described by $\boldsymbol{\theta}_{0}$ or not. The subspace-based detection algorithm, a solution to that problem proposed in Basseville et al. (2000, 2004), builds on a residual associated with the output-only covariance-driven subspace-based identification algorithm.

This algorithm consists in computing the singular value decomposition of

$$
\hat{\mathcal{H}}_{p+1, q}^{0} \quad \operatorname{def}\left(\begin{array}{cccc}
\hat{R}_{0}^{0} & \hat{R}_{1}^{0} & \vdots & \hat{R}_{q-1}^{0} \\
\hat{R}_{1}^{0} & \hat{R}_{2}^{0} & \vdots & \hat{R}_{q}^{0} \\
\vdots & \vdots & \vdots & \vdots \\
\hat{R}_{p}^{0} & \hat{R}_{p+1}^{0} & \vdots & \hat{R}_{p+q-1}^{0}
\end{array}\right)
$$

(the empirical Hankel matrix filled with correlations computed on reference data corresponding to the undamaged structure state). Let $\boldsymbol{\theta}_{0}$ be the identified parameter. It can be characterized as follows (Basseville et al. 2000). Let $\mathcal{O}_{p+1}(\boldsymbol{\theta})$ be the observability matrix written in the modal basis, namely

$$
\mathcal{O}_{p+1}(\boldsymbol{\theta})=\left[\begin{array}{l}
\Phi \\
\Phi \Delta \\
\vdots \\
\Phi \Delta^{p}
\end{array}\right]
$$

def

where $\Delta=\operatorname{diag}(\boldsymbol{\Lambda})$ and $\boldsymbol{\Lambda}$ and $\Phi$ are as in Eq. (5). Then, if the orthonormal matrix $S$ is in the null space of the observability matrix associated with the reference condition, that is

$$
S^{T} \mathcal{O}_{p+1}\left(\boldsymbol{\theta}_{0}\right)=0
$$

then it must also be in the null space of the Hankel matrix filled with correlations of the data taken from the reference model, namely

$$
S^{T} \hat{\mathcal{H}}_{p+1, q}^{0}=0
$$

Thus $\hat{\mathcal{H}}$ and $\mathcal{O}$ should have the same left null space. Note that matrix $S$ in Eq. (8) depends implicitly on $\boldsymbol{\theta}$ and is not unique; nevertheless it can be treated as a function $S(\boldsymbol{\theta})$ (Basseville et al. 2000).

Let $\hat{\mathcal{H}}_{p+1, q}$ be the empirical Hankel matrix filled with correlations of new data from the (possibly damaged) system. To check whether a damage has occurred, in other words if the new data are still well described by the reference parameter $\boldsymbol{\theta}_{0}$, the idea is to check whether this new matrix $\hat{\mathcal{H}}_{p+1, q}$ has the same left null space. For this purpose, a subspace-based residual is defined as

$$
\boldsymbol{\zeta}_{n}\left(\boldsymbol{\theta}_{0}\right)=\sqrt{n} \operatorname{vec}\left[S\left(\boldsymbol{\theta}_{0}\right)^{T} \hat{\mathcal{H}}_{p+1, q}\right]
$$

where $n=$ sample size.

\section{$\chi^{2}$ Test for Global Modal Monitoring}

Testing if $\boldsymbol{\theta}=\boldsymbol{\theta}_{0}$ holds true or not, or equivalently deciding that the residual $\zeta_{n}\left(\boldsymbol{\theta}_{0}\right)$ is significantly different from zero, requires the probability distribution of $\boldsymbol{\zeta}_{n}\left(\boldsymbol{\theta}_{0}\right)$ which is generally unknown. A possible approach to circumvent this situation is the statistical local approach on which tests between the two hypotheses are assumed to be close to each other, namely

$$
\mathbf{H}_{0}: \boldsymbol{\theta}=\boldsymbol{\theta}_{0} \quad \text { and } \quad \mathbf{H}_{1}: \boldsymbol{\theta}=\boldsymbol{\theta}_{0}+\boldsymbol{\delta} \boldsymbol{\theta} / \sqrt{n}
$$

where vector $\boldsymbol{\delta} \boldsymbol{\theta}$ is unknown, but fixed. For large $n$, hypothesis $\mathbf{H}_{1}$ corresponds to small deviations in $\boldsymbol{\theta}$. Define the mean sensitivity: $\mathcal{J}\left(\boldsymbol{\theta}_{0}\right)=-1 / \sqrt{n} \partial /\left.\partial \boldsymbol{\theta} \mathbf{E}_{\boldsymbol{\theta}_{0}} \zeta_{n}(\boldsymbol{\theta})\right|_{\boldsymbol{\theta}=\boldsymbol{\theta}_{0}}$ and the covariance matrix: $\Sigma\left(\boldsymbol{\theta}_{0}\right)=\lim _{n \rightarrow \infty} \mathbf{E}_{\boldsymbol{\theta}_{0}}\left(\zeta_{n} \zeta_{n}^{T}\right)$. Then, provided that $\Sigma\left(\boldsymbol{\theta}_{0}\right)$ is positive definite, and for all $\boldsymbol{\delta} \boldsymbol{\theta}$, the residual $\zeta_{n}\left(\boldsymbol{\theta}_{0}\right)$ in Eq. (10) is asymptotically Gaussian distributed under both hypotheses in Eq. (11) on $\boldsymbol{\theta}$ (Basseville 1998), that is

$$
\zeta_{n}\left(\boldsymbol{\theta}_{0}\right) \underset{n \rightarrow \infty}{\longrightarrow} \mathcal{N}\left[\mathcal{J}\left(\boldsymbol{\theta}_{0}\right) \boldsymbol{\delta} \boldsymbol{\theta}, \Sigma\left(\boldsymbol{\theta}_{0}\right)\right]
$$

Thus a deviation $\boldsymbol{\delta} \boldsymbol{\theta}$ in the system parameter $\boldsymbol{\theta}$ is reflected into a change in the mean value of the residual $\zeta_{n}\left(\boldsymbol{\theta}_{0}\right)$. Matrices $\mathcal{J}\left(\boldsymbol{\theta}_{0}\right)$ and $\Sigma\left(\boldsymbol{\theta}_{0}\right)$, which depend on neither $n$ nor $\boldsymbol{\delta} \boldsymbol{\theta}$, can be estimated prior to testing, using data on the reference system. Consistent estimates $\hat{\mathcal{J}}\left(\boldsymbol{\theta}_{0}\right)$ and $\hat{\Sigma}\left(\boldsymbol{\theta}_{0}\right)$ of $\mathcal{J}\left(\boldsymbol{\theta}_{0}\right)$ and $\Sigma\left(\boldsymbol{\theta}_{0}\right)$ write as follows (Basseville et al. 2004; Zhang and Basseville 2003):

$$
\hat{\mathcal{J}}\left(\boldsymbol{\theta}_{0}\right)=\left[I_{q r} \otimes S\left(\boldsymbol{\theta}_{0}\right)^{T}\right]\left[\hat{\mathcal{H}}_{p+1, q}^{0 T} \mathcal{O}_{p+1}^{\dagger}\left(\boldsymbol{\theta}_{0}\right)^{T} \otimes I_{(p+1) r}\right] \mathcal{O}_{p+1}^{\prime}\left(\boldsymbol{\theta}_{0}\right)
$$

$$
\begin{aligned}
& \mathcal{O}_{p+1}^{\prime}\left(\boldsymbol{\theta}_{0}\right) \\
& =\left(\begin{array}{ccc|cc}
\Lambda_{1}^{\prime(p)} \otimes \varphi_{1} & & 0 & \Lambda_{1}^{(p)} \otimes I_{r} & \\
& \ddots & & & \\
0 & & \Lambda_{m}^{\prime(p)} \otimes \varphi_{m} & & \\
0 & & \Lambda_{m}^{(p)} \otimes I_{r}
\end{array}\right) \\
& \Lambda_{i}^{(p) T}=\left(\begin{array}{lll}
1 & \lambda_{i} \lambda_{i}^{2} \ldots \lambda_{i}^{p}
\end{array}\right), \quad 1 \leqslant i \leqslant m \\
& \Lambda_{i}^{\prime(p) T}=\left(\begin{array}{lll}
0 & 1 & 2 \lambda_{i} \ldots p \lambda_{i}^{p-1}
\end{array}\right), \quad 1 \leqslant i \leqslant m \\
& \hat{\Sigma}\left(\theta_{0}\right)=1 / K \ell \sum_{k=1}^{K} \zeta_{k} \zeta_{k}^{T}, \quad n \approx K \ell
\end{aligned}
$$

with residual $\zeta_{k}$ computed using $Y_{(k-1) \ell+1}, \ldots, Y_{k \ell}$ 
Assume that $\mathcal{J}\left(\boldsymbol{\theta}_{0}\right)$ is full column rank. [Typically, the rank of $\mathcal{J}(\boldsymbol{\theta})$ is equal to the dimension of $\boldsymbol{\theta}$, namely the number of modes plus the number of mode shapes times the number of sensors.] Then, deciding whether residual $\zeta_{n}\left(\boldsymbol{\theta}_{0}\right)$ is significantly different from zero, stated as testing between the two hypotheses in Eq. (11), can be achieved with the aid of the generalized likelihood ratio (GLR) test. The GLR test computes the log-likelihood ratio of the distributions of the residual $\zeta_{n}\left(\boldsymbol{\theta}_{0}\right)$ under each of the hypotheses in Eq. (11). Since both distributions are Gaussian, as displayed in Eq. (12), the GLR test in that case writes

$$
\begin{aligned}
\sup _{\boldsymbol{\delta} \boldsymbol{\theta} \neq 0}\{ & \left.-\left[\zeta_{n}\left(\boldsymbol{\theta}_{0}\right)-\hat{\mathcal{J}}\left(\boldsymbol{\theta}_{0}\right) \boldsymbol{\delta} \boldsymbol{\theta}\right]^{T} \hat{\Sigma}^{-1}\left(\boldsymbol{\theta}_{0}\right)\left[\zeta_{n}\left(\boldsymbol{\theta}_{0}\right)-\hat{\mathcal{J}}\left(\boldsymbol{\theta}_{0}\right) \boldsymbol{\delta} \boldsymbol{\theta}\right]\right\} \\
& +\zeta_{n}\left(\boldsymbol{\theta}_{0}\right) \hat{\Sigma}^{-1}\left(\boldsymbol{\theta}_{0}\right) \zeta_{n}\left(\boldsymbol{\theta}_{0}\right)
\end{aligned}
$$

This boils down to the following $\chi^{2}$-test statistics:

$$
\begin{aligned}
\chi_{n}^{2}\left(\boldsymbol{\theta}_{0}\right)= & \zeta_{n}^{T}\left(\boldsymbol{\theta}_{0}\right) \hat{\Sigma}^{-1}\left(\boldsymbol{\theta}_{0}\right) \hat{\mathcal{J}}\left(\boldsymbol{\theta}_{0}\right) \\
& \times\left[\mathcal{J}^{T}\left(\boldsymbol{\theta}_{0}\right) \hat{\Sigma}^{-1}\left(\boldsymbol{\theta}_{0}\right) \hat{\mathcal{J}}\left(\boldsymbol{\theta}_{0}\right)\right]^{-1} \hat{\mathcal{J}}^{T}\left(\boldsymbol{\theta}_{0}\right) \hat{\Sigma}^{-1}\left(\boldsymbol{\theta}_{0}\right) \zeta_{n}\left(\boldsymbol{\theta}_{0}\right)
\end{aligned}
$$

which should be compared to a threshold. In what follows, the above $\mathcal{J}$ and $\Sigma$ and the index $n$ when clear from context are dropped for simplicity.

The test $\chi_{n}^{2}\left(\boldsymbol{\theta}_{0}\right)$ is asymptotically $\chi^{2}$ distributed with $\operatorname{rank}(\mathcal{J})$ degrees of freedom and noncentrality parameter $\delta \boldsymbol{\theta}^{T} \mathbf{F}\left(\boldsymbol{\theta}_{0}\right) \boldsymbol{\delta} \boldsymbol{\theta}$, where $\mathbf{F}\left(\boldsymbol{\theta}_{0}\right)$ is the asymptotic Fisher information on $\boldsymbol{\theta}_{0}$ contained in $\zeta_{n}\left(\boldsymbol{\theta}_{0}\right)$

$$
\mathbf{F}\left(\boldsymbol{\theta}_{0}\right) \stackrel{\text { def }}{=} \mathcal{J}\left(\boldsymbol{\theta}_{0}\right)^{T} \Sigma\left(\boldsymbol{\theta}_{0}\right)^{-1} \mathcal{J}\left(\boldsymbol{\theta}_{0}\right)
$$

\section{$\chi^{2}$ Tests for Focused Monitoring}

Focusing the monitoring on some subsets of components of the parameter vector $\boldsymbol{\theta}$ is of interest for many purposes. For example, it may be desirable to monitor a specific mode $\lambda$ and the associated mode shape $\varphi_{\lambda}$. For this purpose, and without loss of generality, let $\boldsymbol{\theta}, \mathcal{J}$, and $\mathbf{F}$ be partitioned as

$$
\begin{aligned}
\boldsymbol{\theta} & =\left(\begin{array}{c}
\boldsymbol{\theta}_{a} \\
\boldsymbol{\theta}_{b}
\end{array}\right), \quad \mathcal{J}=\left(\begin{array}{ll}
\mathcal{J}_{a} & \mathcal{J}_{b}
\end{array}\right), \quad \mathbf{F}=\left(\begin{array}{ll}
\mathbf{F}_{a a} & \mathbf{F}_{a b} \\
\mathbf{F}_{b a} & \mathbf{F}_{b b}
\end{array}\right) \\
& =\left(\begin{array}{ll}
\mathcal{J}_{a}^{T} \Sigma^{-1} \mathcal{J}_{a} & \mathcal{J}_{a}^{T} \Sigma^{-1} \mathcal{J}_{b} \\
\mathcal{J}_{b}^{T} \Sigma^{-1} \mathcal{J}_{a} & \mathcal{J}_{b}^{T} \Sigma^{-1} \mathcal{J}_{b}
\end{array}\right)
\end{aligned}
$$

For deciding between $\boldsymbol{\delta} \boldsymbol{\theta}_{a}=0$ and $\boldsymbol{\delta} \boldsymbol{\theta}_{a} \neq 0$, two approaches may be used (Basseville 1997).

The sensitivity approach assumes $\boldsymbol{\theta}_{b}=0$ and consists in projecting the deviations in $\boldsymbol{\delta} \boldsymbol{\theta}$ onto the subspace generated by the components $\boldsymbol{\delta} \boldsymbol{\theta}_{a}$. This results in $\widetilde{\chi}_{a}^{2}=\widetilde{\zeta}_{a}^{T} \mathbf{F}_{a a}^{-1} \widetilde{\zeta}_{a}$, where

$$
\widetilde{\zeta}_{a}^{\text {def }}=\mathcal{J}_{a}^{T} \Sigma^{-1} \zeta
$$

is the partial residual. Note that $\tilde{\chi}_{a}^{2}$ might be sensitive to a change in $\boldsymbol{\theta}_{b}$ which is a limitation in the actual focusing capability of that test and that, for $a \neq b$, residuals $\widetilde{\zeta}_{a}$ and $\widetilde{\zeta}_{b}$ are correlated (Basseville 1997).

The min-max approach consists in viewing parameter $\boldsymbol{\delta} \boldsymbol{\theta}_{b}$ as a nuisance, and rejecting it by replacing it in the likelihood with its least favorable value. This results in the test

$$
\chi_{a}^{\star 2}=\zeta_{a}^{\star T} \mathbf{F}_{a}^{\star-1} \zeta_{a}^{\star}
$$

where the robust residual $\zeta_{a}^{\star}$ is defined by

$$
\zeta_{a}^{\star}=\tilde{\zeta}_{a}-\mathbf{F}_{a b} \mathbf{F}_{a b}^{-1} \tilde{\zeta}_{b}
$$

and where $\mathbf{F}_{a}^{\star}=\mathbf{F}_{a a}-\mathbf{F}_{a b} \mathbf{F}_{b b}^{-1} \mathbf{F}_{b a}$. Note that $\zeta_{a}^{\star}$ is the residual from the regression of the partial residual $\tilde{\zeta}_{a}$ in $\boldsymbol{\delta} \boldsymbol{\theta}_{a}$ on the partial residual $\widetilde{\zeta}_{b}$ in $\boldsymbol{\delta} \boldsymbol{\theta}_{b}$ (nuisance). Under both hypotheses in Eq. (11), test statistics $\chi_{a}^{\star 2}$ is $\chi^{2}$ distributed with $l_{a}$ degrees of freedom and noncentrality parameter $\boldsymbol{\delta} \boldsymbol{\theta}_{a}^{T} \mathbf{F}_{a}^{\star} \boldsymbol{\delta} \boldsymbol{\theta}_{a}$ under $\boldsymbol{\delta} \boldsymbol{\theta}_{a} \neq 0$ and for all $\boldsymbol{\delta} \boldsymbol{\theta}_{b}$. This property makes the residual $\zeta_{a}^{\star}$ and the associated $\chi^{2}$-test $\chi_{a}^{\star 2}$ good candidates for performing the desired monitoring focused on $\boldsymbol{\theta}_{a}$.

\section{Thermal Effect Modeling}

We now introduce the key steps in modeling the temperature effect on the laboratory test case used in the application section. Assuming an Euler-Bernoulli beam and neglecting rotating inertia, the eigenproblem governing the vibrations of a clamped planar beam, axially prestressed, is given by the following equations (Géradin and Rixen 1997):

$$
\left\{\begin{array}{l}
E I \frac{d^{4} \mathbf{w}(x)}{d x^{4}}-N_{0} \frac{d^{2} \mathbf{w}(x)}{d x^{2}}-\rho A \omega^{2} \mathbf{w}(x)=0 \\
\left.\mathbf{w}(x)\right|_{x=0, L}=0 ;\left.\quad \frac{d \mathbf{w}(x)}{d x}\right|_{x=0, L}=0
\end{array}\right.
$$

Here $\omega=$ eigenpulsation and $\mathbf{w}(x)=$ corresponding transversal displacement. The relation with the discrete-time mode and mode shape in Eq. (4) and continuous-time mode in Eq. (2) is

$$
\begin{gathered}
\omega=\frac{1}{\tau} \arctan \frac{\Im(\lambda)}{\mathfrak{R}(\lambda)}=\Im(\mu) \\
\mathbf{w}=\mathbf{w}_{\omega}=\boldsymbol{\phi}_{\lambda}
\end{gathered}
$$

In Eq. (21), $E, I, \rho$, and $A$ denote Young's modulus, the inertia momentum of the cross section, the density, and cross-sectional area, respectively (all assumed constant). $N_{0}=$ quasistatic axial preload given by

$$
\begin{gathered}
N_{0}=E A \varepsilon(x) \\
\varepsilon(x)=\varepsilon_{0}(x)-\alpha \delta T, \quad \delta T=T_{0}(x)-T_{\text {ref }}
\end{gathered}
$$

In Eq. (24), $\varepsilon_{0}=$ mechanical strain; $T_{0}=$ current temperature; $T_{\text {ref }}=$ reference temperature for which there is no stress (corresponding to the temperature just before the tightening beam ends); and $\alpha$ denotes the thermal expansion coefficient. It is important to note that $N_{0}$ remains spatially constant-even though a temperature gradient might exist along the beam-because no external axial body forces nor axial surface tractions are present during the experiments (in particular, gravity effects are negligible). Thus, only one measure at a given point is necessary. This is obtained by thermally compensated strain gauges, yielding a direct measure of $\varepsilon(x)=N_{0} / E A$.

\section{Updating the Modal Parameters}

It can be shown that the eigenproblem in Eq. (21) yields an infinite number of solutions for $\omega_{\ell}(\ell=1, \ldots, \infty)$, given by the characteristic equation (Bokaian, 1988) 


$$
\begin{aligned}
& 2 \gamma_{\ell}^{-} \gamma_{\ell}^{+}\left[1-\cos \left(\gamma_{\ell}^{-} L\right) \cosh \left(\gamma_{\ell}^{+} L\right)\right]+\left(\gamma_{\ell}^{+2}-\gamma_{\ell}^{-2}\right) \sin \left(\gamma_{\ell}^{-} L\right) \sinh \left(\gamma_{\ell}^{+} L\right) \\
& \quad=0
\end{aligned}
$$

with the following notation:

$$
\gamma_{\ell}^{ \pm}=\left[\sqrt{\frac{\rho A}{E I} \omega_{\ell}^{2}+\left(\frac{N_{0}}{2 E I}\right)^{2}} \pm \frac{N_{0}}{2 E I}\right]^{1 / 2}
$$

Eq. (25) must be solved numerically because no analytical solutions are available for the clamped case. The mode shapes are then given by

$$
\begin{aligned}
w_{\ell}(x)= & C_{\ell}\left[\left(\cos \gamma_{\ell}^{-} x-\cosh \gamma_{\ell}^{+} x\right)\right. \\
& \left.-\frac{\cos \gamma_{\ell}^{-} L-\cosh \gamma_{\ell}^{+} L}{\gamma_{\ell}^{+} \sin \gamma_{\ell}^{-} L-\gamma_{\ell}^{-} \sinh \gamma_{\ell}^{+} L}\left(\gamma_{\ell}^{+} \sin \gamma_{\ell}^{-} x-\gamma_{\ell}^{-} \sinh \gamma_{\ell}^{+} x\right)\right]
\end{aligned}
$$

where $C_{\ell}=$ normalization constant.

For each value of temperature shift $\delta T, N_{0} / E A$, and thus $\varepsilon(x)$ in Eq. (23), is measured; the eigenpulsations $\omega_{\ell}$ 's are computed from Eqs. (25) and (26) and the mode shapes $w_{\ell}(x)$ 's are computed from Eq. (27). The corresponding temperature-updated modal parameter $\boldsymbol{\theta}_{T}$ is then recovered from Eqs. (22) and (5). This is summarized as follows:

$$
\delta T \rightarrow \varepsilon(x) \rightarrow\left(\omega_{\ell}\right)_{\ell} \rightarrow\left(\lambda_{\ell}, \boldsymbol{\varphi}_{\ell}\right)_{\ell=1, m} \rightarrow \boldsymbol{\theta}_{T}
$$

The number $m$ of identified modal parameters $\left(\lambda_{\ell}, \boldsymbol{\varphi}_{\ell}\right)$ is limited by the number $r$ of sensors and results from experimental to analytical mode matching.

In the study reported in this paper, the mode shapes $w_{\ell}(x)$ 's are assumed to be unaffected by the temperature effect. Thus Formula (27) is not used, and the $\varphi_{\ell}$ 's in Eq. (28) are the mode shapes stacked in $\theta_{0}$.

\section{Computing the Thermal Sensitivities}

The variational formulation associated with the equations in Eq. (21) is written, for any complex number $v$, as

$$
E I \int_{0}^{L} \frac{d^{2} v}{d x^{2}} \frac{d^{2} w}{d x^{2}} d x+N_{0} \int_{0}^{L} \frac{d v}{d x} \frac{d w}{d x} d x-\omega^{2} \rho A \int_{0}^{L} v w d x=0
$$

with $\left.v\right|_{x=0, L}=d v /\left.d x\right|_{x=0, L}=0$, where $L=$ beam length. A temperature change causes a variation $\delta N_{0}$ of the axial prestress, and thus a variation of the modes. The first variation of this formulation writes

$$
\begin{aligned}
& E I \int_{0}^{L} \frac{d^{2} v}{d x^{2}} \frac{d^{2} \delta w}{d x^{2}} d x+\int_{0}^{L}\left(N_{0} \frac{d v}{d x} \frac{d \delta w}{d x}-\omega^{2} \rho A v \delta w+\delta N_{0} \frac{d v}{d x} \frac{d w}{d x}\right) d x \\
& \quad=\delta\left(\omega^{2}\right) \rho A \int_{0}^{L} v w d x
\end{aligned}
$$

From Eq. (29), the sum of the first three terms in the left-hand side of Eq. (30) is equal to zero. Thus, writing Eq. (30) for $v=w=w_{\ell}$, the sensitivity of the eigenfrequency $\omega_{\ell}$ with respect to the axial prestress writes

$$
\delta\left(\omega_{\ell}^{2}\right)=\frac{\int_{0}^{L}\left(d w_{\ell} / d x\right)^{2} d x}{\rho A \int_{0}^{L} w_{\ell}^{2} d x} \delta N_{0}
$$

Knowing the mode shapes $w_{\ell}$, the expression in Eq. (31) can be computed semianalytically.

The sensitivity with respect to the temperature $T$ is then obtained from $\delta N_{0}=-E A \alpha \delta T$, which results from Eqs. (23) and (24). From Eq. (31) and Basseville et al. (2004), it is deduced that the sensitivity of the $\ell$ th component (mode) of the parameter $\boldsymbol{\theta}$ with respect to the temperature $T$ writes

$$
\begin{aligned}
\mathcal{J}_{\boldsymbol{\theta} T}(\ell) & \stackrel{\text { def }}{=} \frac{\partial \lambda_{\ell}}{\partial \mu_{\ell}} \frac{\partial \mu_{\ell}}{\partial \omega_{\ell}} \frac{\partial \omega_{\ell}}{\partial T}=\frac{\partial \lambda_{\ell}}{\partial \mu_{\ell}} \frac{\partial \mu_{\ell}}{\partial \omega_{\ell}} \frac{1}{2 \omega_{\ell}} \frac{\partial \omega_{\ell}^{2}}{\partial T} \\
& =\frac{\tau E \alpha \int_{0}^{L}\left(d w_{\ell} / d x\right)^{2} d x}{2 \omega_{\ell} \rho \int_{0}^{L} w_{\ell}^{2} d x}\left(\begin{array}{c}
\Im(\lambda) \\
-\Re(\lambda)
\end{array}\right)
\end{aligned}
$$

The sensitivity matrix (actually, a vector) of the modal parameter $\boldsymbol{\theta}$ with respect to the temperature $T$ has $m$ components of the Form (32). The other components of $\mathcal{J}_{\boldsymbol{\theta} T}$ are equal to zero since the prestress effects upon the mode shapes are negligible, at least for the temperatures considered during the experiment (see 3), and are thus neglected in what follows. Therefore $\mathcal{J}_{\boldsymbol{\theta} T}$ writes

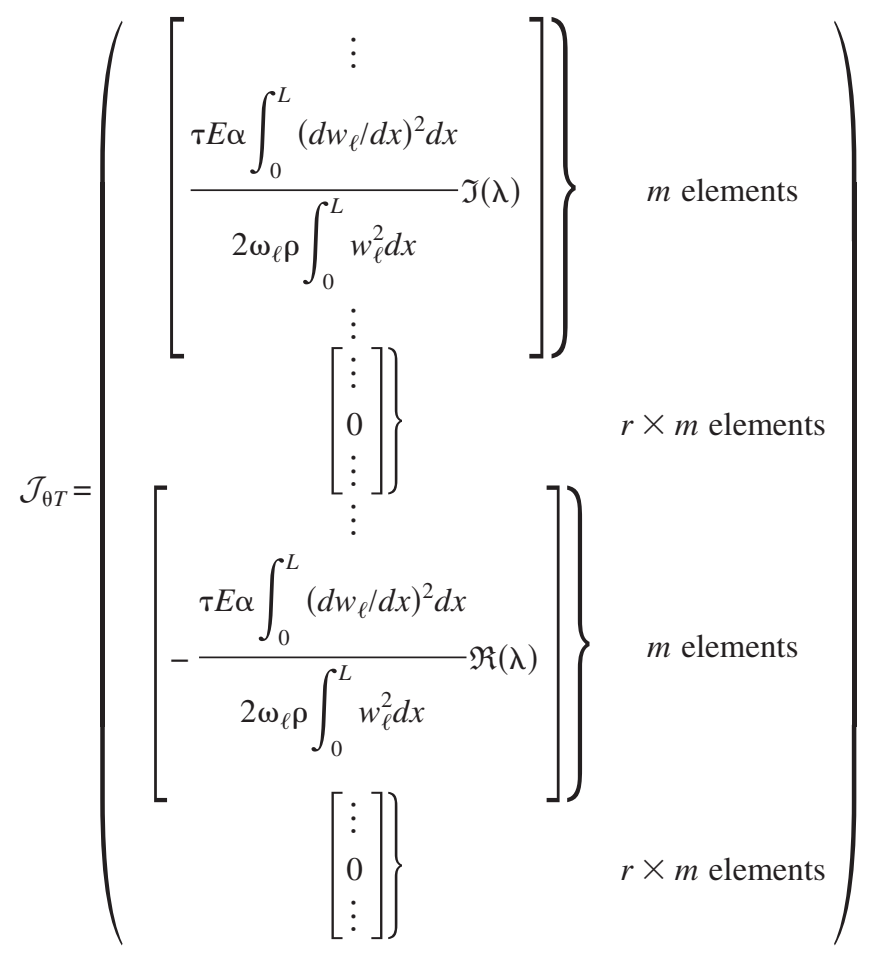

\section{Two Analytical Approaches to Temperature Handling}

In this section, two analytical approaches are proposed for handling the temperature effect in vibration-based SHM within the framework of the subspace-based detection algorithm [Eqs. (10) and (13)-(15)]. The first approach updates the null space $S$ in Eq. (8) to account for possible modal changes due to temperature 
effects. The second approach handles the temperature as a nuisance parameter.

\section{Temperature-Adjusted Null Space Detection}

Having recorded both a new data sample $\left(Y_{k}\right)_{k=1, \ldots, n}$ from the (possibly damaged) system and the corresponding temperature $T$, the idea is to update the modal parameter using Eq. (28), then to compute $\mathcal{O}_{p+1}\left(\boldsymbol{\theta}_{T}\right)$ from Eq. (7) and the adjusted null space $S\left(\boldsymbol{\theta}_{T}\right)$ such that

$$
S^{T}\left(\boldsymbol{\theta}_{T}\right) \mathcal{O}_{p+1}\left(\boldsymbol{\theta}_{T}\right)=0
$$

Then, filling the empirical Hankel matrix [Eq. (6)] with the correlation matrices of the new data, the residual is computed as

$$
\zeta_{n}\left(\boldsymbol{\theta}_{T}\right)=\sqrt{n} \operatorname{vec}\left[S^{T}\left(\boldsymbol{\theta}_{T}\right) \hat{\mathcal{H}}_{p+1, q}\right]
$$

and the proposed temperature-adjusted damage detection test $\chi_{n}^{2}\left(\boldsymbol{\theta}_{T}\right)$ is computed as in Eq. (15).

\section{Rejecting the Temperature Effect Seen as a Nuisance}

The method used in this paper consists in using the material presented above for focused monitoring with the modal parameter $\boldsymbol{\theta}$ and the temperature $T$ playing the role of $\boldsymbol{\theta}_{a}$ and $\boldsymbol{\theta}_{b}$, respectively. More precisely, assuming small deviations in both $\boldsymbol{\theta}$ and $T$, the mean value of the residual $\zeta_{n}$ now writes

$$
\mathbf{E}_{1}\left(\zeta_{n}\right)=\mathcal{J}\left(\boldsymbol{\theta}_{0}\right) \boldsymbol{\delta} \boldsymbol{\theta}+\mathcal{J}_{T} \delta T
$$

where $\mathcal{J}\left(\boldsymbol{\theta}_{0}\right)$ is as in Eq. (12), $\mathcal{J}_{T}$ is defined as

$$
\mathcal{J}_{T}^{\text {def }}=\left.\mathcal{J}\left(\boldsymbol{\theta}_{0}\right) \mathcal{J}_{\boldsymbol{\theta} T}\right|_{\boldsymbol{\theta}_{0}}
$$

and where the matrix $\left.\mathcal{J}_{\boldsymbol{\theta} T}\right|_{\boldsymbol{\theta}_{0}}$ contains the sensitivities of the modes with respect to the temperature, computed at $\boldsymbol{\theta}_{0}$ from Eq. (33). Because of the expression for $\mathcal{J}_{T}$, computing the $\chi^{2}$ test in Eq. (19) associated with the robust residual [Eq. (20)] requires, in addition to $\mathcal{J}\left(\boldsymbol{\theta}_{0}\right)$, the computation of $\left(\left.\mathcal{J}_{\boldsymbol{\theta} T}\right|_{\boldsymbol{\theta}_{0}}\right)$.

\section{Application}

The global test [Eq. (15)] and the two new algorithms of the previous section have been experimented on a vertical clamped beam equipped with accelerometers and installed within a climatic chamber, a laboratory test case provided by Laboratoire Central des Ponts et Chaussées.

\section{Description of the Test Case}

The experimental device is depicted in Fig. 1. A vertical beam is clamped at both ends on a workbench made of four vertical thick columns and two horizontal decks. This workbench is made of steel, whereas the beam is made of aluminum. The whole apparatus is set inside a climatic chamber with controlled ambient temperature. Because steel and aluminum have different thermal expansion coefficients $\left(11.7 \times 10^{-6}\right.$ and $23.4 \times 10^{-6} \mathrm{~K}^{-1}$, respectively), a temperature change naturally induces a significant axial prestress, constant along the beam in the absence of external forces.

The test beam is instrumented with four accelerometers, located at nodes of the fifth flexural mode in order to avoid nodes of Modes 1-4 [a truncation up to the fourth mode has been
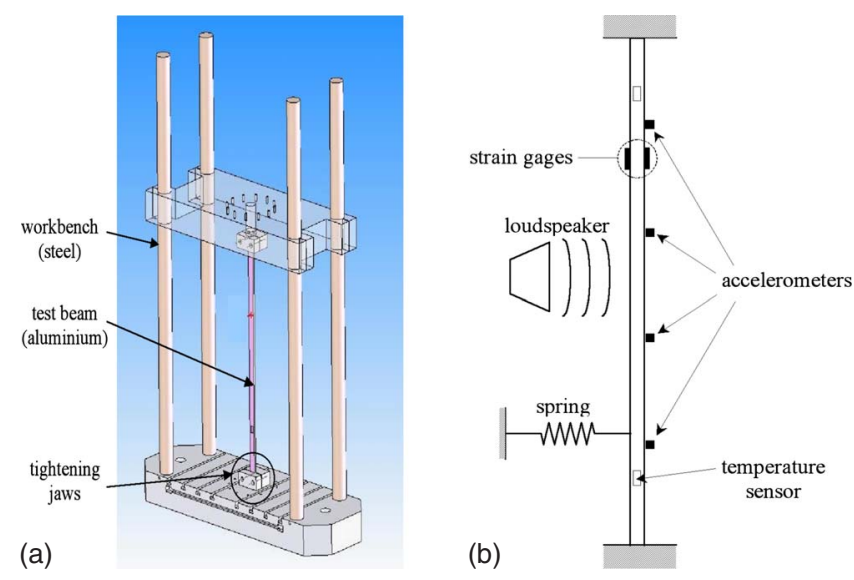

Fig. 1. Workbench and instrumented test beam

performed in the analysis reported in Basseville et al. (2006)]. A pair of aluminum strain gauges with thermal compensation is also bonded on the beam. The half sum of both strains gives a direct measure of the actual axial prestress (divided by Young's modulus), without requiring any temperature measurement. This measurement is of primary importance for the method using a temperature-adjusted null space. Some temperature sensors have also been used in order to check that gauge measurements are closely related to thermal variations.

Tests are carried out inside the climatic chamber, first by stabilizing the ambient temperature for $1 \mathrm{~h}$ and then cooling down for $17 \mathrm{~h}$ with a slope of $-1^{\circ} \mathrm{C} / \mathrm{h}$. The beam is acoustically excited by a loudspeaker with a white noise input. Strains and temperature measurements are saved every second. Acceleration measurements are automatically triggered every $30 \mathrm{~min}$ for $600 \mathrm{~s}$ with a sampling frequency of $1,280 \mathrm{~Hz}$, which is sufficient for Modes 1-4 (the fourth frequency is below $500 \mathrm{~Hz}$ ).

An example of experimental results is displayed in Fig. 2. It clearly shows that prestress and thus frequencies increase as temperature decreases with time (note that prestress is taken positive when tensile). At the end of the test, the first frequency has been increased by about $16 \%$. Modes 2, 3, and 4 (not shown here) have been increased by about 8,5 , and $3 \%$, respectively. Axial prestress has thus a stronger effect for lower frequencies, which is consistent with known results (Bokaian 1988). As displayed in Fig. 3, the effect of the axial prestress on the first four mode

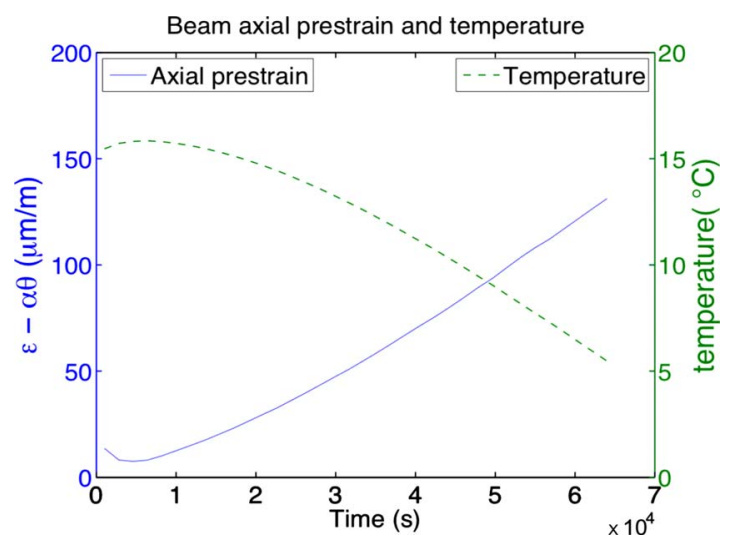

Fig. 2. Time variations of the axial prestress and temperature 

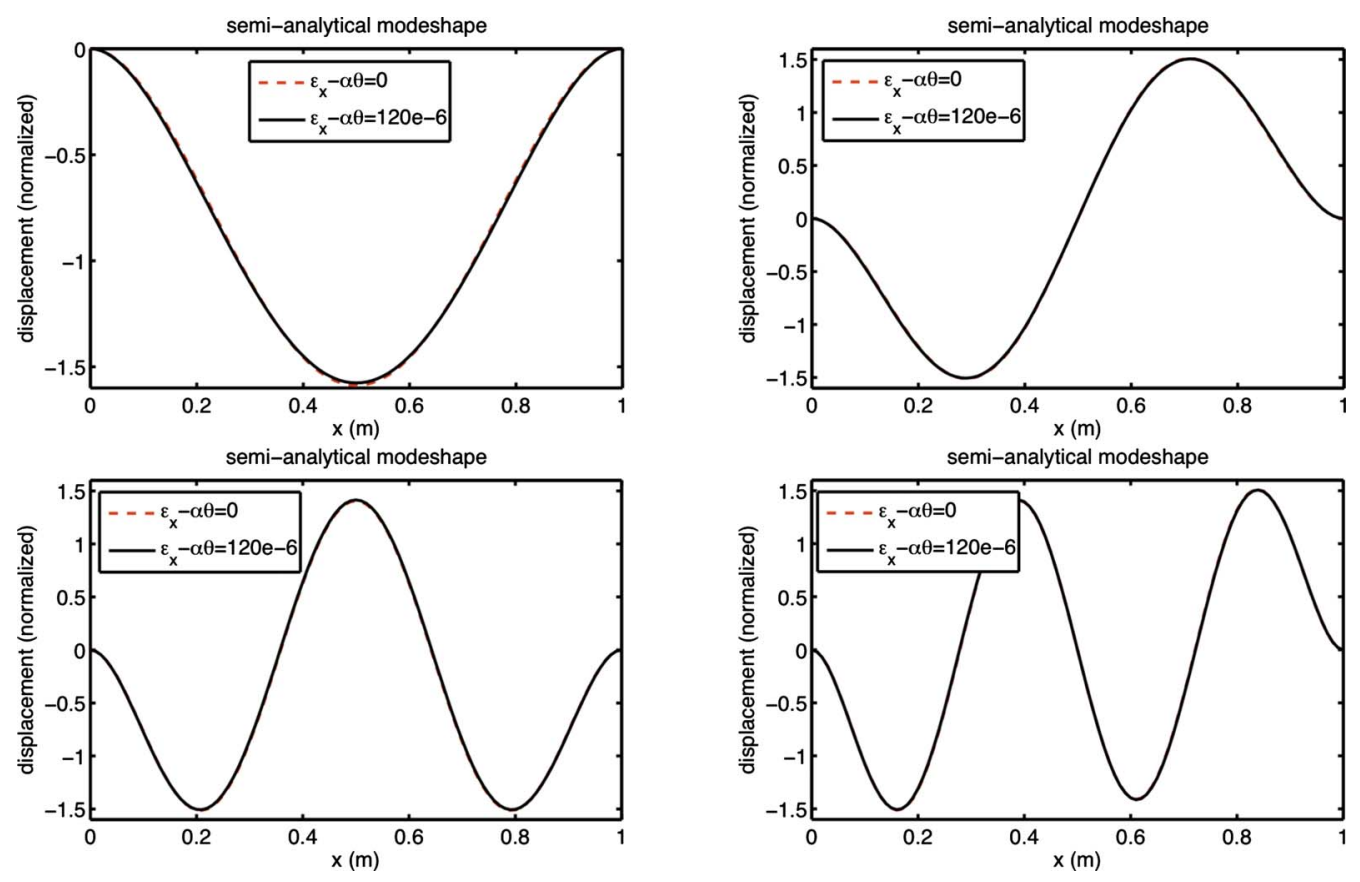

Fig. 3. Temperature effect on the first four mode shapes

shapes is hardly visible, and thus negligible, at least for the temperatures considered during the experiment. Hence the assumption made above on mode shapes unaffected by the prestress is valid in the present case. As for the damping coefficients, it is well known that they are subject to a larger uncertainty than the frequencies (Gersch 1974). It may happen that this uncertainty encompasses the temperature effects, but no knowledge is available about that.

Finally, two sets of experiments are performed, for the safe and damaged cases, respectively. In each set, 37 temperature scenarios are undertaken, and each scenario is repeated 10 times (one scenario every $30 \mathrm{~min}$ ). The effect of a horizontal clamped spring attached to the beam plays the role of a local nondestructive damage. Indeed, the presence of the spring slightly modifies the frequencies, as a damage would do, causing an additional offset that is nearly constant throughout the experiment. This damage may be tuned by increasing the stiffness and/or the spring attachment point. In this experiment, the spring is located at $L / 5$ and its stiffness is about $k=4,000 \mathrm{~N} \cdot \mathrm{m}^{-1}$. This choice has been designed from a finite-element model in order to give slight deviations (less than 1\%) from the no-spring (safe) case. Note that the finite-element model used is simplified as a beam-spring system (the workbench is not accounted for). Experimental deviations of Modes 1-4 are about $+0.8,+0.4,-0.1$, and $-0.2 \%$, respectively. Though weak, the deviations for Modes 3 and 4 are slightly negative. This is probably due to the mechanical coupling of the beam with the spring bracket, which has a non-negligible flexibility compared to the workbench (this bracket is connected to the workbench and is not accounted for either in the finiteelement model). Fig. 4 shows how the first four frequencies are affected by the temperature scenarios, under both safe (blue) and damaged (red) conditions. In that theory, the prebending effects have been neglected upon dynamics, which is acceptable for experiments because cooling has been chosen instead of heating in order to avoid any prebuckling bending.

\section{Implementation Issues}

Some comments are in order on the selection of the key parameters and the computation of the key matrices for each of the two proposed methods. The reference parameter vector $\boldsymbol{\theta}_{0}$, the null space $S\left(\boldsymbol{\theta}_{0}\right)$ in Eq. (8), and the key matrices $\hat{\mathcal{J}}\left(\boldsymbol{\theta}_{0}\right)$ in Eq. (13) and $\hat{\Sigma}\left(\boldsymbol{\theta}_{0}\right)$ in Eq. (14) for the global test [Eqs. (10) and (15)] are estimated on the 10 realizations of the first scenario corresponding to a thermal constraint approximately equal to $20 \mu \mathrm{m} / \mathrm{m}$. The computations in Eqs. (28) and (33), for the modal updating and sensitivities, respectively, have been made with the following values of beam thickness and length: $h=0.01 \mathrm{~m}$ and $L=1 \mathrm{~m}$ (depth: $b=0.03 \mathrm{~m}$ ). Young's modulus and density are $E=6.95 e+10 \mathrm{~Pa}$ and $\rho=2,700 \mathrm{~kg} \cdot \mathrm{m}^{-3}$. Those characteristics have been experimentally checked with static bending tests and also by comparing theoretical and experimental free eigenfrequencies.

\section{Temperature-Adjusted Subspace Detection}

Based on the temperature measurement for the current scenario, the adjusted parameter $\boldsymbol{\theta}_{T}$ in Eq. (28) can be computed using the analytical model [Eqs. (25)-(27)]. Since the mode shapes are assumed constant, this provides us with the evolution with respect to $\varepsilon(x)$ of the frequencies only.

In the experiment, the clamped boundary condition-obtained with tightening jaws-is not perfect, which explains a negative offset in the experimental frequencies compared with theory (less than 3\%). As displayed in Fig. 4, the computed and identified first frequencies are different, under both safe and damaged conditions, respectively. Comparing the black plots with respect to the corresponding blue ones, the frequencies in Eq. (28) appear slightly biased, and the accuracy of $\boldsymbol{\theta}_{T}$ can only be assumed in the vicinity of $\boldsymbol{\theta}_{0}$. For this reason, based on an identified reference parameter $\widetilde{\boldsymbol{\theta}}_{0}$, a modified parameter vector $\widetilde{\boldsymbol{\theta}}_{T}$ is computed, which contains mode shape values, damping coefficients, and unbiased 

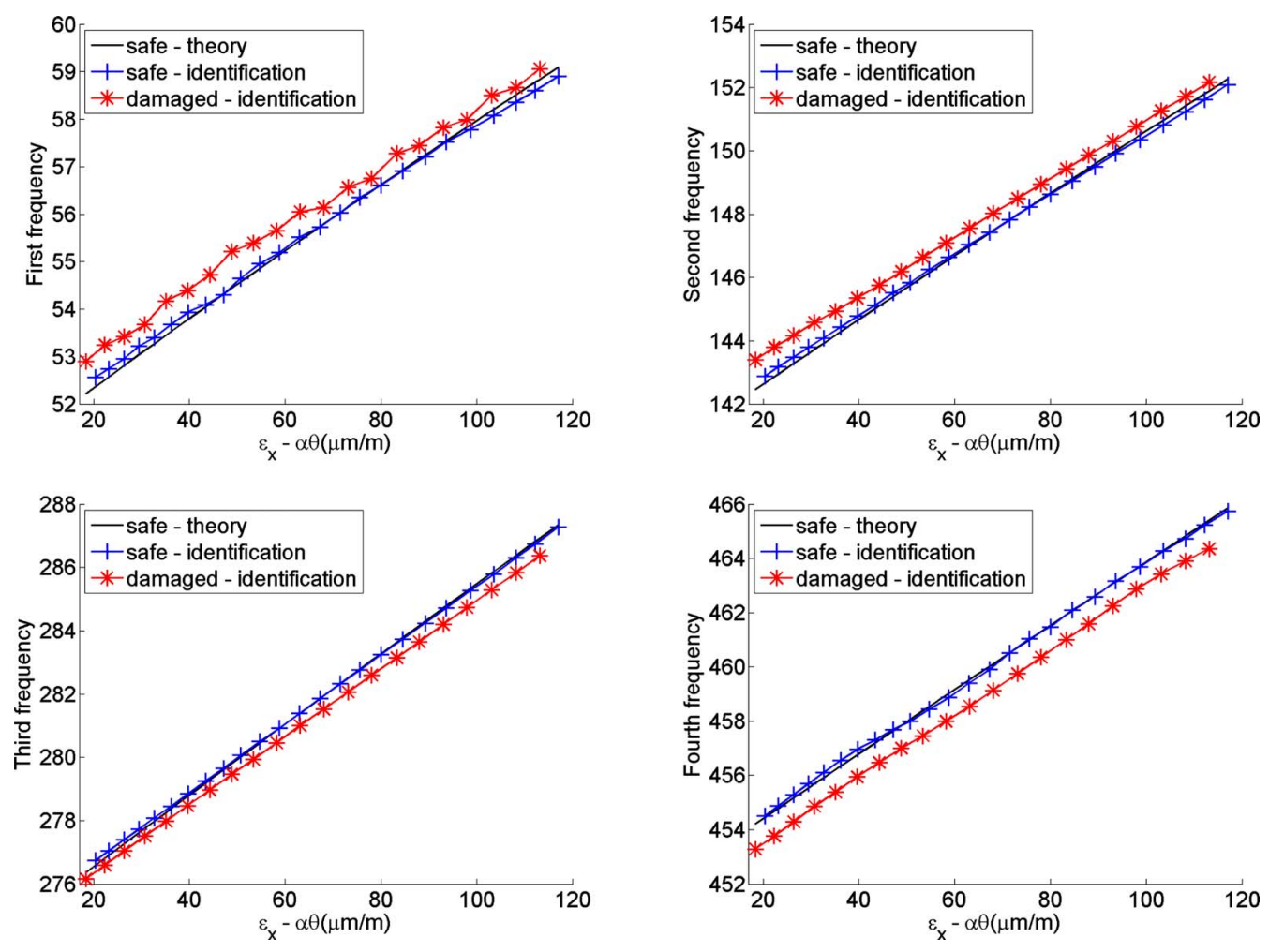

Fig. 4. Temperature effect on the four frequencies in both safe and damaged cases

frequencies. Actually, assuming that the slope of the black plots in Fig. 4 is correct, a simple shift helps getting rid of the bias

$$
(\forall \varepsilon) \overline{\boldsymbol{\theta}}_{T}(\varepsilon)=\boldsymbol{\theta}_{T}(\varepsilon)+\overline{\boldsymbol{\theta}}_{0}\left(\varepsilon_{0}\right)-\boldsymbol{\theta}_{T}\left(\varepsilon_{0}\right)
$$

The proposed algorithm for the temperature-adjusted test based on the residual in Eq. (35) runs as follows. Having at hand a reference modal parameter $\boldsymbol{\theta}_{0}$ for the safe structure, and having recorded an $n$-size sample of new measurements $\left(Y_{k}\right)$, namely for every realization of every temperature scenario:

- Compute the adjusted parameter vector $\boldsymbol{\theta}_{T}$ in Eq. (28) and update it into $\widetilde{\boldsymbol{\theta}}_{T}$ from Eq. (38);

- Compute the observability matrix $\mathcal{O}\left(\tilde{\boldsymbol{\theta}}_{T}\right)$ from Eq. (7) and the null space $S\left(\widetilde{\boldsymbol{\theta}}_{T}\right)$ from Eq. (34);

- Compute the Hankel matrix in Eq. (6) and the residual in Eq. (35), namely $\boldsymbol{\zeta}_{n}\left(\widetilde{\boldsymbol{\theta}}_{T}\right)=\sqrt{n} \operatorname{vec}\left[S^{T}\left(\widetilde{\boldsymbol{\theta}}_{T}\right) \hat{\mathcal{H}}\right]$;

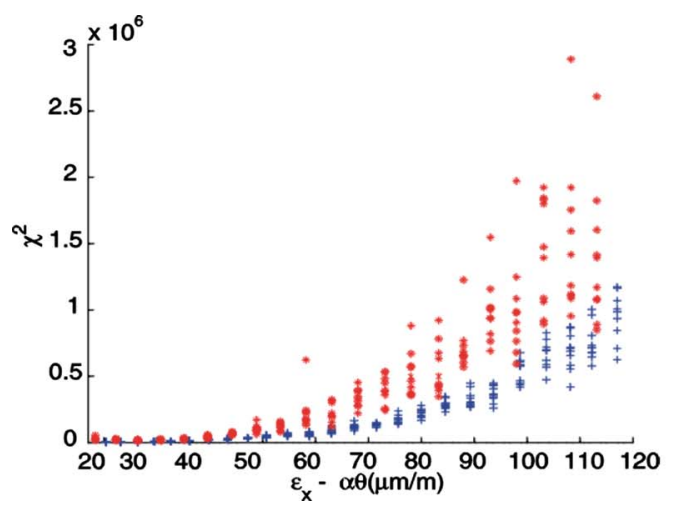

- Compute the Jacobian matrix $\hat{\mathcal{J}}\left(\widetilde{\boldsymbol{\theta}}_{T}\right)$ as in Eq. (13) and the covariance matrix $\hat{\Sigma}\left(\widetilde{\boldsymbol{\theta}}_{T}\right)$ as in Eq. (14); and

- Compute the $\chi^{2}$ test:

$$
\begin{aligned}
\chi_{n}^{2}\left(\widetilde{\boldsymbol{\theta}}_{T}\right)= & \boldsymbol{\zeta}_{n}^{T}\left(\widetilde{\boldsymbol{\theta}}_{T}\right) \hat{\Sigma}^{-1}\left(\widetilde{\boldsymbol{\theta}}_{T}\right) \hat{\mathcal{J}}\left(\tilde{\boldsymbol{\theta}}_{T}\right) \\
& \times\left[\hat{\mathcal{J}}^{T}\left(\widetilde{\boldsymbol{\theta}}_{T}\right) \hat{\Sigma}^{-1}\left(\widetilde{\boldsymbol{\theta}}_{T}\right) \hat{\mathcal{J}}\left(\widetilde{\boldsymbol{\theta}}_{T}\right)\right]^{-1} \hat{\mathcal{J}}^{T}\left(\widetilde{\boldsymbol{\theta}}_{T}\right) \hat{\Sigma}^{-1}\left(\widetilde{\boldsymbol{\theta}}_{T}\right) \boldsymbol{\zeta}_{n}\left(\widetilde{\boldsymbol{\theta}}_{T}\right)
\end{aligned}
$$

\section{Temperature Rejection}

For each value of temperature shift $\delta T, N_{0} / E A$, and thus $\varepsilon(x)$ in Eq. (23), is measured, and the $\delta\left(\omega_{\ell}^{2}\right)$ 's are computed from Eq. (31). The proposed algorithm runs as follows. Having at hand a long data sample for the safe structure and a reference modal parameter vector $\boldsymbol{\theta}_{0}$ identified on that data sample:

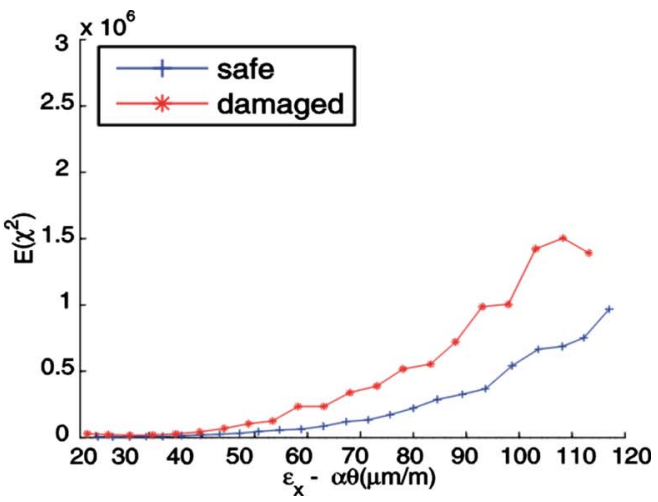

Fig. 5. Global $\chi^{2}$ test [Eq. (15)] 

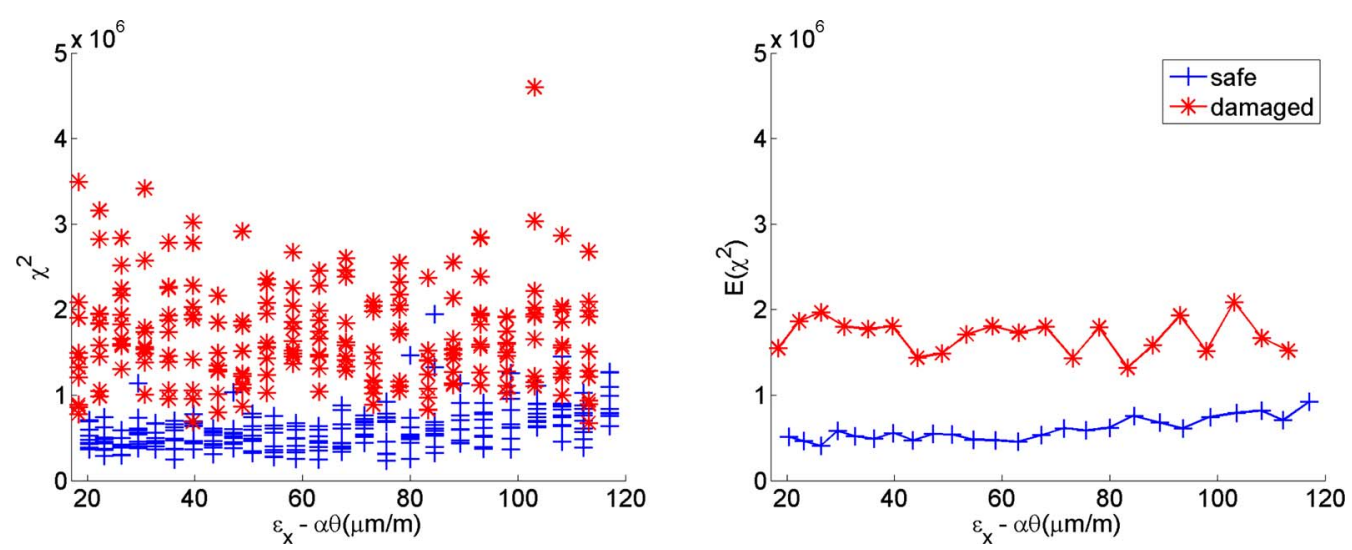

Fig. 6. $\chi^{2}$ test [Eq. (39)] with temperature-adjusted null space

- Compute the observability matrix $\mathcal{O}\left(\boldsymbol{\theta}_{0}\right)$ from Eq. (7) and the null space $S\left(\boldsymbol{\theta}_{0}\right)$ from Eq. (8);

- Compute the sensitivities with respect to $\boldsymbol{\theta}_{0}$ and $T$, namely $\hat{\mathcal{J}}\left(\boldsymbol{\theta}_{0}\right)$ in Eq. (13), $\left.\mathcal{J}_{\boldsymbol{\theta} T}\right|_{\boldsymbol{\theta}_{0}}$ from Eq. (33), and $\mathcal{J}_{T}$ in Eq. (37);

- Compute the covariance $\hat{\Sigma}\left(\boldsymbol{\theta}_{0}\right)$ in Eq. (14), and Fisher matrix in Eq. (16) for the entire parameter vector: and $\mathbf{F}=\mathbf{F}\left(\boldsymbol{\theta}_{0}, T\right)$.

Then, having recorded an $n$-size sample of new measurements

$\left(Y_{k}\right)$, namely for every realization of every temperature scenario:

- Compute the Hankel matrix in Eq. (6) and the residual in Eq. (10), namely $\boldsymbol{\zeta}_{n}\left(\boldsymbol{\theta}_{0}\right)=\sqrt{n} \operatorname{vec}\left[S^{T}\left(\boldsymbol{\theta}_{0}\right) \hat{\mathcal{H}}\right]$;

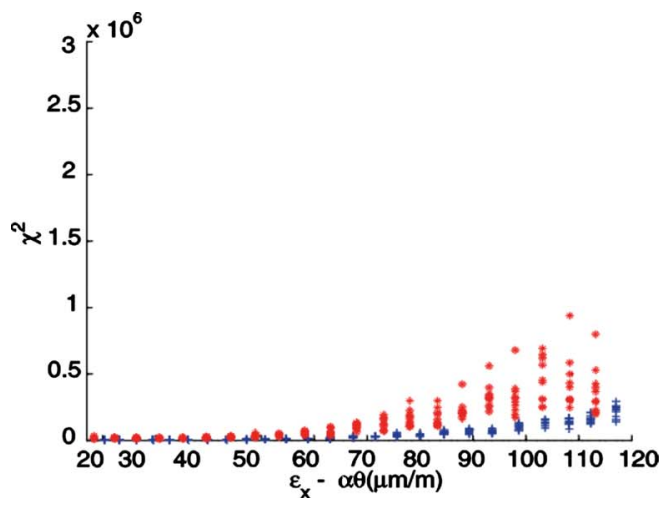

- Compute the robust residual as in Eq. (20), namely $\boldsymbol{\zeta}_{\boldsymbol{\theta}_{0}}^{\star}=\widetilde{\zeta}_{\boldsymbol{\theta}_{0}}$ $-\mathbf{F}_{\boldsymbol{\theta}_{0}, T} \mathbf{F}_{T, T}^{-1} \tilde{\zeta}_{T}$, where the partial residuals $\tilde{\boldsymbol{\zeta}}_{\boldsymbol{\theta}_{0}}, \widetilde{\boldsymbol{\zeta}}_{T}$ are computed as in Eq. (18) and $\mathbf{F}_{\boldsymbol{\theta}_{0}, T}, \mathbf{F}_{T, T}$ are submatrices of $\mathbf{F}$ as in Eq. (17); and

- Compute the min-max $\chi^{2}$ test as in Eq. (19), namely

$$
\chi_{n}^{\star 2}\left(\boldsymbol{\theta}_{0}\right)=\zeta_{\boldsymbol{\theta}_{0}}^{\star T} \mathbf{F}_{\boldsymbol{\theta}_{0}}^{\star-1} \zeta_{\boldsymbol{\theta}_{0}}^{\star}
$$

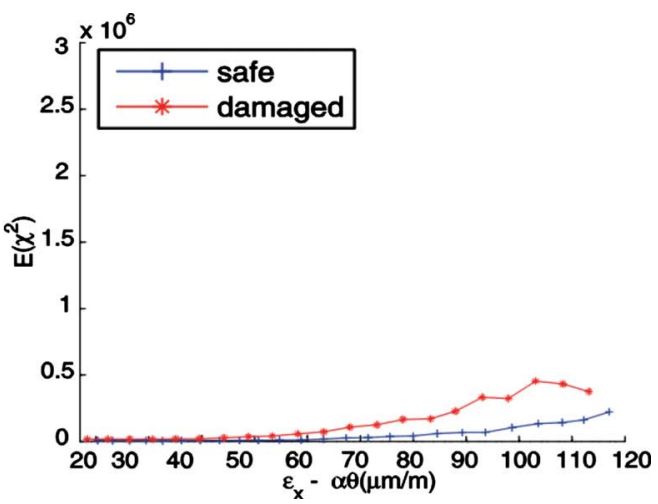

Fig. 7. $\chi^{2}$ test [Eq. (40)] with temperature rejection
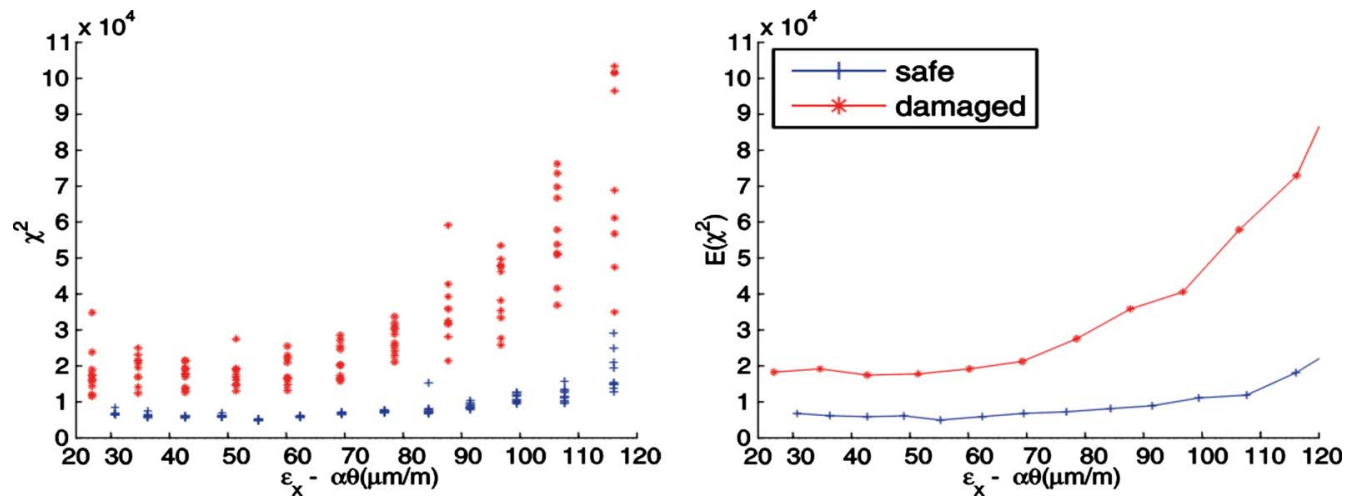

Fig. 8. $\chi^{2}$ test [Eq. (40)]—Fig. 7 zoomed near $\theta_{0}$ 

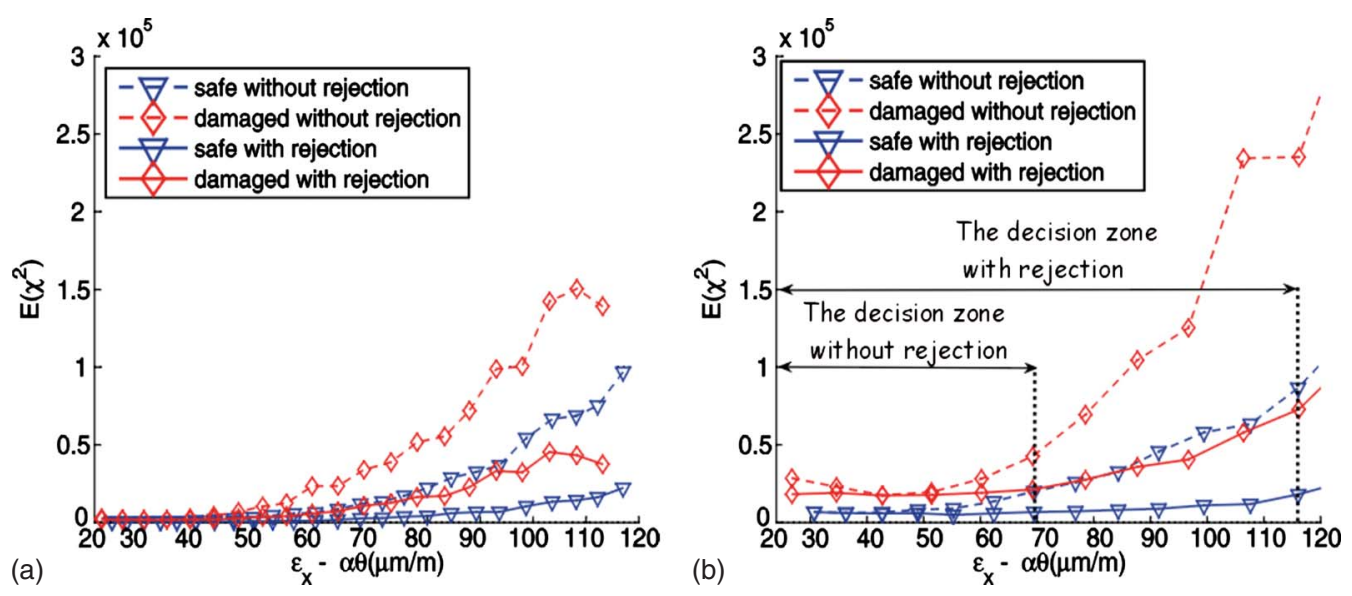

Fig. 9. Comparing Fig. 5 (right) and Fig. 7 (right)

\section{Numerical Results}

The results obtained on this test case with the global test [Eq. (15)] and the two new algorithms [Eqs. (39) and (40)] are displayed in Figs. 5-9 for the safe (blue) and damaged (red) cases. For all these figures, the test values on the $Y$-axis are plotted against the thermal constraint on the $X$-axis. Figs. 5-7 correspond to 25 scenarios with thermal constraints ranging approximately between 20 and $115 \mu \mathrm{m} / \mathrm{m}$, and Fig. 8 corresponds to 11 scenarios with thermal constraints ranging between 20 and $65 \mu \mathrm{m} / \mathrm{m}$. In all but the last figure, the left part displays the test values for the 10 repetitions of the 25 temperature scenarios, while the test values averaged over the 10 realizations of the same scenario are displayed in the right part. It should be obvious that 10 is too small a number for clearing out possible outliers; this is confirmed in Fig. 6 (right). The values of the global subspacebased $\chi^{2}$ test in Eq. (15) are displayed in Fig. 5.

\section{Temperature-Adjusted Subspace Detection}

In Fig. 6, the values of the test [Eq. (39)] are displayed. Whereas in Fig. 5 (left), it is not possible to discriminate between the damaged and the undamaged values for the test [Eq. (15)]; in Fig. 6 (left) it is possible to set a threshold for separating the test values in the damaged and safe scenarios. Fig. 6 (right) provides us with the same information about the dispersion of the test values, which are much higher in the damaged case, as theoretically predicted. For the plots in Fig. 6 (right), $\widetilde{\boldsymbol{\theta}}_{0}$ has been estimated on scenarios corresponding to the middle value $\varepsilon(x)=71.497 \mu \mathrm{m} / \mathrm{m}$. It should be noticed that the choice of $\widetilde{\boldsymbol{\theta}}_{0}$ for computing $\widetilde{\boldsymbol{\theta}}_{T}$ influences the behavior of the detection test.

\section{Temperature Rejection}

The values of the min-max $\chi^{2}$ test [Eq. (40)] are displayed in Fig. 7 for all the scenarios. Fig. 8 displays a zoom near the upper values of the reference scenario $\boldsymbol{\theta}_{0}$ corresponding to a prestrain of $\varepsilon(x)-\alpha \boldsymbol{\theta}=20.344 \mu \mathrm{m} / \mathrm{m}$. A better visualization of the comparative capabilities and limitations of the min-max $\chi^{2}$ test [Eq. (40)] with respect to the global $\chi^{2}$ test [Eq. (15)] is achieved in Fig. 9. In Fig. 9 (left), it appears that the min-max test is smaller than the global one, as expected, and thus less sensitive to small increases in $\varepsilon(x)$. In Fig. 9 (right), it is seen that damage detection can be achieved for larger values of $\varepsilon$ when using the min-max test than when using the global one. Actually, the range for $\varepsilon(x)$ where the tests values under safe conditions can be separated from the val- ues under damaged conditions extends from $40 \mu \mathrm{m} / \mathrm{m}$ for the global test to $65 \mu \mathrm{m} / \mathrm{m}$ for the min-max test. Fig. 9 (right) also shows that the nuisance rejection is only local, as predicted by the small deviation hypothesis, and fails when $\varepsilon(x)$ too much deviates from the nominal value.

\section{Comparison with Global Empirical Null Space Detection}

The parametric algorithm proposed in this paper has been compared to a nonparametric approach to temperature handling which consists in using an empirical null space (Balmès et al. 2008). The following comments are in order. The two methods differ in two ways. In this paper, a parametric kernel is generated from a model, whereas for the nonparametric approach the kernel is learned from a collection of reference scenarios. The parametric kernel is expected to yield lower values for the safe scenario. The drawback is that one must know the value of $\varepsilon(x)$ for each scenario, but there is no need for a training period. The second difference between the two methods is that the residual covariance is here computed for each scenario, and only once for the nonparametric method. The present approach is thus computationally more expensive. But it is also more robust with respect to changes in the excitation.

\section{Conclusions}

The problem of handling the temperature effect in vibration-based monitoring of civil engineering structures has been addressed. Two analytical approaches have been proposed, based on a subspace-based damage detection algorithm previously proposed by some of the writers and on a model of the temperature effect on the modal parameters. The first approach is based on a temperature-adjusted null space plugged into that damage detection algorithm. In the second approach, the temperature is seen as a nuisance effect, and the thermal model is used together with a statistical rejection approach to nuisance parameter handling. The relevance of the proposed algorithms has been illustrated on a laboratory test case within a climatic chamber.

The model of the temperature effect on the modal parameters considered in this article involves only an axial prestress, which is relevant for the application example. For more complex structures, thermal prestress in all directions should be taken into ac- 
count and the model should be extended to a three-dimensional temperature field. In such a case, a relevant parameterization for the thermal model [here Eqs. (23) and (24)] should be selected, and the problem of handling the thermal parameters as nuisance parameters becomes more involved. Another issue for future research is the validation on structures in-operation.

\section{Acknowledgments}

This work was carried out within the Project No. 2003-54 CONSTRUCTIF, coordinated by IRISA, in the framework of the French Computer and Security program (ACI S\&I). Preliminary versions of parts of this paper were presented at the 3rd European Workshop on Structural Health Monitoring, Granada, S., July 2006 for the temperature-adjusted null space method, and at the 4th World Conference on Structural Control and Monitoring, San Diego, July 2006 for the temperature rejection method.

\section{Notation}

The following symbols are used in this paper:

$$
\begin{aligned}
& A=\text { cross-sectional area; } \\
& C=\text { damping matrix; } \\
& E=\text { Young's modulus; } \\
& \mathbf{E}=\text { expectation operator; } \\
& \mathbf{F}=\text { Fisher information matrix; } \\
& F, H=2 m \times 2 m \text { state transition and } r \times 2 m \\
& \text { observation matrices; } \\
& f=\text { frequency (discrete-time model); } \\
& \mathbf{H}_{0}=\text { null hypothesis for } \boldsymbol{\theta} \text {; } \\
& \mathbf{H}_{1}=\text { alternative hypothesis for } \boldsymbol{\theta} \text {; } \\
& \mathcal{H}_{p, q}=\text { Hankel matrix, with } p \text { block rows and } q \\
& \text { block columns; } \\
& I=\text { inertia momentum of the beam cross section; } \\
& \mathcal{J}=\text { sensitivity matrix of } \zeta \text { with respect to } \boldsymbol{\delta} \boldsymbol{\theta} \text {; } \\
& K, M=\text { stiffness and mass matrices; } \\
& L=\text { beam length; } \\
& N_{0}=\text { quasi-static axial preload (in newton); } \\
& \mathcal{O}_{p}=\text { observability matrix, with } p \text { block rows; } \\
& R_{i}=\operatorname{lag} i \text { correlation matrix of } Y \text {; } \\
& S=(p+1) \cdot r \text { orthogonal matrix; } \\
& T=\text { temperature; } \\
& V_{k}, X_{k}=\text { discrete-time state noise and state vector; } \\
& w=\text { transversal displacement associated with } \omega \text {; } \\
& Y_{k}=\text { discrete-time measurement vector; } \\
& \mathcal{Z}(t)=\text { vector collecting the displacements of the } \\
& \text { degrees of freedom of the structure; } \\
& \alpha=\text { thermal expansion coefficient; } \\
& \Delta, \Lambda=\text { diagonal matrix and vector, respectively, filled } \\
& \text { with the } \lambda \text { 's; } \\
& \boldsymbol{\delta} \boldsymbol{\theta}=\text { deviation in } \boldsymbol{\theta} \\
& \varepsilon(x)=\text { strain; } \\
& \boldsymbol{\zeta}_{n}, \tilde{\boldsymbol{\zeta}}_{a}, \boldsymbol{\zeta}_{a}^{\star}=\text { residual, partial residual, and robust residual }
\end{aligned}
$$

$$
\begin{aligned}
\boldsymbol{\phi}_{\lambda}, \boldsymbol{\varphi}_{\lambda}= & \text { eigenvector of } F \text { associated with } \lambda \text { and its } \\
& \text { observed components; } \\
\chi_{n}^{2}, \tilde{\chi}_{a}^{2}, \chi_{a}^{\star 2}= & \text { chi-square tests associated with } \zeta_{n}, \tilde{\zeta}_{a}, \zeta_{a}^{*}, \\
& \text { respectively; } \\
\Phi= & \text { matrix whose columns are the } \boldsymbol{\varphi}_{\lambda} \text { 's; } \\
\Psi_{\mu}= & \text { mode shape associated with mode } \mu ; \\
\omega= & \text { eigenpulsation ln } \lambda ; \text { and } \\
= & \hat{A} \text { : estimated value of a scalar, vector, or } \\
& \text { matrix } A .
\end{aligned}
$$

\section{References}

Alampalli, S. (2000). "Effects of testing, analysis, damage and environment on modal parameters." Mech. Syst. Signal Process., 14(1), 6374.

Alvandi, A., and Cremona, C. (2006). "Assessment of vibration-based damage identification techniques." J. Sound Vib., 292(1-2), 179-202.

Balmès, E., Basseville, M., Bourquin, F., Nasser, H., and Treyssède, F. (2008). "Merging sensor data from multiple temperature scenarios for vibration-based monitoring of civil structures." Struct. Health Monit., 7(2), 129-142.

Basseville, M. (1997). "Information criteria for residual generation and fault detection and isolation." Automatica, 33(5), 783-803.

Basseville, M. (1998). "On-board component fault detection and isolation using the statistical local approach." Automatica, 34(11), 1391-1416.

Basseville, M., Abdelghani, M., and Benveniste, A. (2000). "Subspacebased fault detection algorithms for vibration monitoring." Automatica, 36(1), 101-109.

Basseville, M., Bourquin, F., Mevel, L., Nasser, H., and Treyssède, F. (2006). "Handling the temperature effect in SHM: Combining a subspace-based statistical test and a temperature-adjusted null space." Proc., European Workshop on Structural Health Monitoring, DEStech Publications Inc., Granada, Spain, 759-766.

Basseville, M., Mevel, L., and Goursat, M. (2004). "Statistical modelbased damage detection and localization: Subspace-based residuals and damage-to-noise sensitivity ratios." J. Sound Vib., 275(3-5), 769-794.

Basseville, M., Mevel, L., Vecchio, A., Peeters, B., and van der Auweraer, H. (2003). "Output-only subspace-based damage detectionApplication to a reticular structure." Struct. Health Monit., 2(2), 161168.

Benveniste, A., and Mevel, L. (2007). "Nonstationary consistency of subspace methods." IEEE Trans. Autom. Control, 52(6), 974-984.

Bokaian, A. (1988). "Natural frequencies of beams under compressive axial loads." J. Sound Vib., 126(1), 49-65.

Catbas, F., Brown, D., and Aktan, A. (2004). "Parameter estimation for multiple-input multiple-output modal analysis of large structures." J. Eng. Mech., 130(8), 921-930.

Cornwell, P., Farrar, C., Doebling, S., and Sohn, H. (1999). "Environmental variability of modal properties." Exp. Tech., 39(6), 45-48.

Deraemaeker, A., Reynders, E., De Roeck, G., and Kullaa, J. (2008). "Vibration-based structural health monitoring using output-only measurements under changing environment." Mech. Syst. Signal Process., 22(1), 34-56.

Ewins, D. (2000). Modal testing: Theory, practice and applications, 2nd Ed., Research Studies, Letchworth, Hertfordshire, U.K.

Farrar, C., Doebling, S., and Nix, D. (2001). "Vibration-based structural damage identification." Philos. Trans. R. Soc. London, Ser. A, 359(1778), 131-150.

Fritzen, C.-P. (2005). "Recent developments in vibration-based structural health monitoring." Proc., Int. Workshop on Structural Health Monitoring, Stanford Univ., Palo Alto, Calif., 42-60.

Fritzen, C.-P., Mengelkamp, G., and Guemes, A. (2003). "Elimination of temperature effects on damage detection within a smart structures concept." Proc., Int. Workshop on Structural Health Monitoring, Stan- 
ford Univ., Palo Alto, Calif., 1530-1538.

Gao, Y., Spencer, B., and Bernal, D. (2007). "Experimental verification of the flexibility-based damage locating vector method." J. Eng. Mech., 133(10), 1043-1049.

Géradin, M., and Rixen, D. (1997). Mechanical vibrations-Theory and application to structural dynamics, 2nd Ed., Wiley, New York.

Gersch, W. (1974). "On the achievable accuracy of structural parameter estimates." J. Sound Vib., 34(1), 63-79.

Golinval, J., Yan, A.-M., Peeters, B., and De Roeck, G. (2004). “A comparative study on damage detection of Z24-Bridge: One-year monitoring with varying environmental conditions." Proc., European Workshop on Structural Health Monitoring, DEStech Publications Inc., Munich, Germany, 791-799.

Juang, J. (1994). Applied system identification, Prentice-Hall, Englewood Cliffs, N.J.

Kullaa, J. (2003). "Is temperature measurement essential in structural health monitoring?" Proc., Int. Workshop on Structural Health Monitoring, Stanford Univ., Palo Alto, Calif., 717-724.

Kullaa, J. (2004). "Structural health monitoring under variable environmental or operational conditions." Proc., European Workshop on Structural Health Monitoring, DEStech Publications Inc., Munich, Germany, 1262-1269.

Mevel, L., Basseville, M., and Goursat, M. (2003a). "Stochastic subspace-based structural identification and damage detectionApplication to the steel-quake benchmark." Mech. Syst. Signal Process., 17(1), 91-101.

Mevel, L., Goursat, M., and Basseville, M. (2003b). "Stochastic subspace-based structural identification and damage detection and localization-Application to the Z24 Bridge benchmark." Mech. Syst. Signal Process., 17(1), 143-151.

Mevel, L., Hermans, L., and van der Auweraer, H. (1999). "Application of a subspace-based fault detection method to industrial structures." Mech. Syst. Signal Process., 13(6), 823-838.

Moorty, S., and Roeder, C. (1992). "Temperature-dependent bridges movements." J. Struct. Eng., 118(4), 1090-1115.

Natke, H., and Cempel, C. (1997). Model-aided diagnosis of mechanical systems: Fundamentals, detection, localization, assessment, Springer, New York.

Pandey, A., and Biswas, M. (1994). "Damage detection in structures using changes in flexibility." J. Sound Vib., 169(1), 3-17.
Peeters, B., et al. (2003). "Online vibration based crack detection during fatigue testing." Key Eng. Mater., 245(2), 571-578.

Peeters, B., and De Roeck, G. (2001). "One year monitoring of the Z24Bridge: Environmental effects versus damage events." Earthquake Eng. Struct. Dyn., 30(2), 149-171.

Peeters, B., Maeck, J., and De Roeck, G. (2001). "Vibration-based damage detection in civil engineering: Excitation sources and temperature effects." Smart Mater. Struct., 10(3), 518-527.

Rohrmann, R., Baessler, M., Said, S., Schmid, W., and Ruecker, W. (2000). "Structural causes of temperature affected modal data of civil structures obtained by long time monitoring." Proc., Int., Modal Analysis Conf.

Sohn, H., Dzwonczyk, M., Straser, E., Kiremidjian, A., Law, K., and Meng, T. (1999). "An experimental study of temperature effect on modal parameters of the Alamosa Canyon Bridge." Earthquake Eng. Struct. Dyn., 28, 879-897.

Steenackers, G., and Guillaume, P. (2005). "Structural health monitoring of the Z24 Bridge in presence of environmental changes using modal analysis." Proc., Int., Modal Analysis Conf., SEM, Orlando, Fla.

Vanlanduit, S., Parloo, E., Cauberghe, B., Guillaume, P., and Verboven, P. (2005). "A robust singular value decomposition for damage detection under changing operating conditions and structural uncertainties." J. Sound Vib., 284(3-5), 1033-1050.

Wood, M. (1992). "Damage analysis of bridge structures using vibrational techniques." Ph.D. thesis, Aston Univ., Birmingham, U.K.

Yan, A.-M., Kerschen, G., De Boe, P., and Golinval, J.-C. (2005a). "Structural damage diagnosis under varying environmental conditions-Part I: A linear analysis." Mech. Syst. Signal Process., 19(4), 847-864.

Yan, A.-M., Kerschen, G., De Boe, P., and Golinval, J.-C. (2005b). "Structural damage diagnosis under varying environmental conditions-Part II: Local PCA for non-linear cases." Mech. Syst. Signal Process., 19(4), 865-880.

Zhang, Q., and Basseville, M. (2003). "Advanced numerical computation of $\chi^{2}$-tests for FDI." Proc., IFAC Symp. SAFEPROCESS, IFAC, Washington, 211-216.

Zang, C., Friswell, M., and Imregun, M. (2007). "Structural health monitoring and damage assessment using frequency response correlation criteria." J. Eng. Mech., 133(9), 981-993. 